\title{
The natural history of severe asthma and influences of early risk factors: a population-based cohort study
}

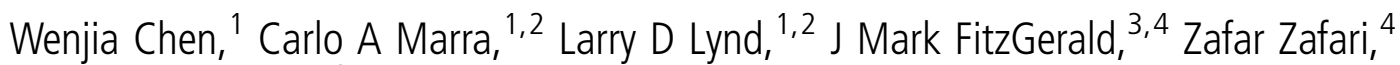 \\ Mohsen Sadatsafavi ${ }^{3,4}$
}

- Additional material is published online only. To view please visit the journal online (http://dx.doi.org/10.1136/ thoraxjn-2015-207530)

${ }^{1}$ Collaboration for Outcomes Research and Evaluation, Faculty of Pharmaceutical Sciences, University of British Columbia, Vancouver, British Columbia, Canada

${ }^{2}$ Centre for Health Evaluation and Outcome Sciences, University of British Columbia, Vancouver, British Columbia, Canada

${ }^{3}$ Department of Medicine, Institute for Heart and Lung Health, University of British Columbia, Vancouver, British Columbia, Canada

${ }^{4}$ Centre for Clinical

Epidemiology and Evaluation, University of British Columbia, Vancouver, British Columbia, Canada

Correspondence to Dr Mohsen Sadatsafavi, Centre for Clinical Epidemiology and Evaluation, 7th Floor, 828

West 10th Avenue, Research Pavilion, Vancouver, BC, Canada V5Z 1M9:

msafavi@mail.ubc.ca

Received 7 July 2015 Revised 10 November 2015 Accepted 25 November 2015 Published Online First 5 January 2016

\section{ABSTRACT}

Background Severe asthma is associated with disproportionately high morbidity, but little is known about its natural history and how risk factors at first year of diagnosis modify its subsequent development. Methods Using administrative health data, we retrospectively followed patients 14-55 years of age with newly diagnosed severe asthma in British Columbia, Canada. Based on intensity of resource use (drug therapy) and occurrence of exacerbations, each patientyear was classified into mild, moderate, or severe asthma. We estimated the probability of transition between severity levels or to death over the study period using a four-state Markov model, and used this to assess the 10-year trajectory of severe asthma and the influence of baseline risk factors.

Results We followed 13467 patients. Ten years after incident severe asthma, $83 \%$ had transitioned to a less severe level (mild: 43\%, moderate: 40\%). Low socioeconomic status, high comorbidity burden, and high adherence (proportion of days covered (PDC) by asthma controller therapy) in the first year were independently associated with, respectively, 10\%, 24\% and 35\% more time in severe asthma over the next 10 years. Sex was not associated with the clinical course.

Conclusions Most patients with incident severe asthma used fewer resources over time, indicating a long-term transition to milder asthma. Potentially modifiable risk factors for poor prognosis of severe asthma include low socioeconomic status and high comorbidity burden. The association between PDC and future asthma severity is likely due to residual confounding by disease severity.

\section{INTRODUCTION}

Asthma is a prevalent chronic disease of the airways ${ }^{1}$ that imposes a substantial burden on individuals and society. ${ }^{2}$ In particular, severe asthma, while affecting only $5-10 \%$ of the asthma population, is associated with the greatest share of asthma morbidity and economic burden. ${ }^{3}$ Severe asthma is characterised by frequent and severe manifestations which do not respond to, or only respond to, highdose therapy of anti-inflammatory and other controller medications. ${ }^{4}$ Although asthma is generally reversible, and milder cases can be effectively controlled with currently available therapy, the clinical course of severe asthma and its risk factors remain poorly understood. Several studies on the prognosis

\section{Key messages}

\section{What is the key question?}

- What is the long-term natural history of severe asthma and what is the influence of risk factors at diagnosis on the subsequent disease trajectory?

\section{What is the bottom line?}

- Patients with incident severe asthma often transition to less severe asthma over time, but low socioeconomic status and high comorbidity at first year of severe asthma are associated with a considerably longer stay in severe asthma over the next 10 years.

\section{Why read on?}

- This is the first study to characterise the 10-year disease trajectory in patients with patterns of resource use indicating severe asthma and it highlights the potential role of socioeconomic status and general health on the long-term course of severe asthma.

of severe asthma drew samples from specialty clinics and are mainly focused on the decline of lung function. ${ }^{5-7}$ Given the unconfirmed clinical significance of such metrics, ${ }^{8}$ these physiological measures cannot provide the necessary evidence of real-life outcomes of asthma, such as the risk of exacerbations over time.

A traditional approach to assessing the severity of asthma is based on symptom burden and lung function metrics before initiating treatment. ${ }^{8}$ However, in the real world many patients are already receiving various forms of therapy at the time of initial assessment. ${ }^{8}$ Moreover, since these metrics are also markers of suboptimal control, some consider it inappropriate to use them to define severity before using a standardised therapy. ${ }^{9}$ For population-based evaluations, asthma severity can be inferred by correlating levels of severity with the amount of medication required to maintain acceptable control, in combination with the markers of asthma-related adverse events, once the treatment has been initiated. ${ }^{8} 10$ The levels of severity measured from these resource-use records was found to correlate well with lung function 
measures, risk of asthma-related hospitalisations, and fatal exacerbations. $^{11} 12$

Despite the high burden, the long-term history of severe asthma and its determinants are not well understood. A longitudinal study using administrative health data found that the majority of patients with indicators of severe asthma received less intensive therapy over time. ${ }^{12}$ However, this study did not consider disease remission and relapse because patients were censored once their therapy suggested non-severe asthma. In addition, the study only evaluated age and sex as potential determinants of the three-year course of the disease.

In our study, we aim to describe the long-term natural history of severe asthma and identify early course risk factors that modify the disease prognosis. This study expands on the previous work in multiple dimensions. Specifically, 10-year trajectories were inferred using a rigorous statistical method that utilised all available longitudinal data to quantify the dynamic transitions across severity states and their association with various risk factors.

\section{METHODS}

\section{Data sources}

A provincial health insurance programme provides universal health care coverage to all legal residents of British Columbia (BC), Canada, one of the largest provinces, representing 13\% of the Canadian population (4.4 million as of 2011). ${ }^{13}$ The

Table 1 Characteristics of study population in the index year

\begin{tabular}{|c|c|}
\hline Characteristic & $\begin{array}{l}\text { Patients with severe } \\
\text { asthma }(\mathrm{N}=13 \text { 467) }\end{array}$ \\
\hline Age, median (IQR) & $36.8(26.6,43.8)$ \\
\hline \multicolumn{2}{|l|}{ Sex, N (\%) } \\
\hline Men & $6094(45)$ \\
\hline Women & $7373(55)$ \\
\hline \multicolumn{2}{|c|}{ Socioeconomic status, N (\%) } \\
\hline Low & $6326(47)$ \\
\hline Middle & $2649(20)$ \\
\hline High & $4492(33)$ \\
\hline \multicolumn{2}{|l|}{ Comorbidity, N (\%) } \\
\hline $\mathrm{CCl}=0$ & $1969(15)$ \\
\hline $\mathrm{CCl}=1$ & $10345(77)$ \\
\hline $\mathrm{CCl}=2$ & $657(5)$ \\
\hline $\mathrm{CCl} \geq 3$ & $496(4)$ \\
\hline \multicolumn{2}{|c|}{ Moderate to severe exacerbation, N (\%) } \\
\hline None & $3262(24)$ \\
\hline$\geq 1$ exacerbation & $10205(76)$ \\
\hline \multicolumn{2}{|c|}{ PDC by asthma controller medications } \\
\hline PDC $<50 \%$ & $8297(62)$ \\
\hline$P D C \geq 50 \%-80 \%$ & $3777(28)$ \\
\hline $\mathrm{PDC} \geq 80 \%$ & $1393(10)$ \\
\hline \multicolumn{2}{|c|}{ Severity in the past year, $\mathrm{N}(\%)$} \\
\hline No asthma* & 1785 (13) \\
\hline Mild & $3674(27)$ \\
\hline Moderate & $8008(59)$ \\
\hline \multicolumn{2}{|c|}{ Severity in the year before last year, $\mathrm{N}(\%)$} \\
\hline No asthma* & 3262 (24) \\
\hline Mild & $4192(31)$ \\
\hline Moderate & $6013(45)$ \\
\hline \multicolumn{2}{|c|}{$\begin{array}{l}\text { * Refers to a patient-year in which the intensity of asthma-related resource utilisation } \\
\text { did not satisfy the case definition of asthma. In the analysis, patients with no asthma } \\
\text { prior to the index year were assumed to be associated with mild asthma. } \\
\text { CCl, Charlson Comorbidity Index; PDC, proportion of days covered. }\end{array}$} \\
\hline
\end{tabular}

administrative demands of this programme have resulted in the creation of centralised databases that capture resource use records for all legal residents, regardless of their third-party insurance coverage or any co-payment. We had access to registration files, ${ }^{14}$ vital statistics, ${ }^{15}$ discharge abstract databases (capturing all instances of hospitalisation), ${ }^{16}$ Medical Services Plan (MSP, capturing outpatient services records) ${ }^{17}$ and PharmaNET (capturing all medications dispensed). ${ }^{18}$ Previous analyses have shown a low prevalence of missing data, under-reporting, and misclassification in these databases. ${ }^{19}$ All databases are linked at the individual level with unique but anonymous identifiers (University of BC Human Ethics Certificate H08-01287) and access permission was granted by the BC Ministry of Health. All inferences, opinions, and conclusions drawn in this research are those of the authors, and do not reflect the opinions or policies of the Data Steward(s). The study period was from 1 January 1997 to 31 December 2012.

\section{Study design and subjects}

We identified patients with asthma using a validated case definition of asthma. ${ }^{2}$ This definition was based on meeting at least one of the following three criteria in any 12-month window within the study period: one or more asthma-related hospitalisation (codes of the International Classification of Diseases 9th edition (ICD-9): 493.x, 10th edition (ICD-10): J45, J46); two or more physician visits with diagnostic ICD codes of asthma; or filling four or more prescriptions for asthma-related medications (see online supplementary appendix 1 for the medication list).

From this initial cohort, we included patients between 14 and 55 years of age with a new onset of severe asthma, identified as
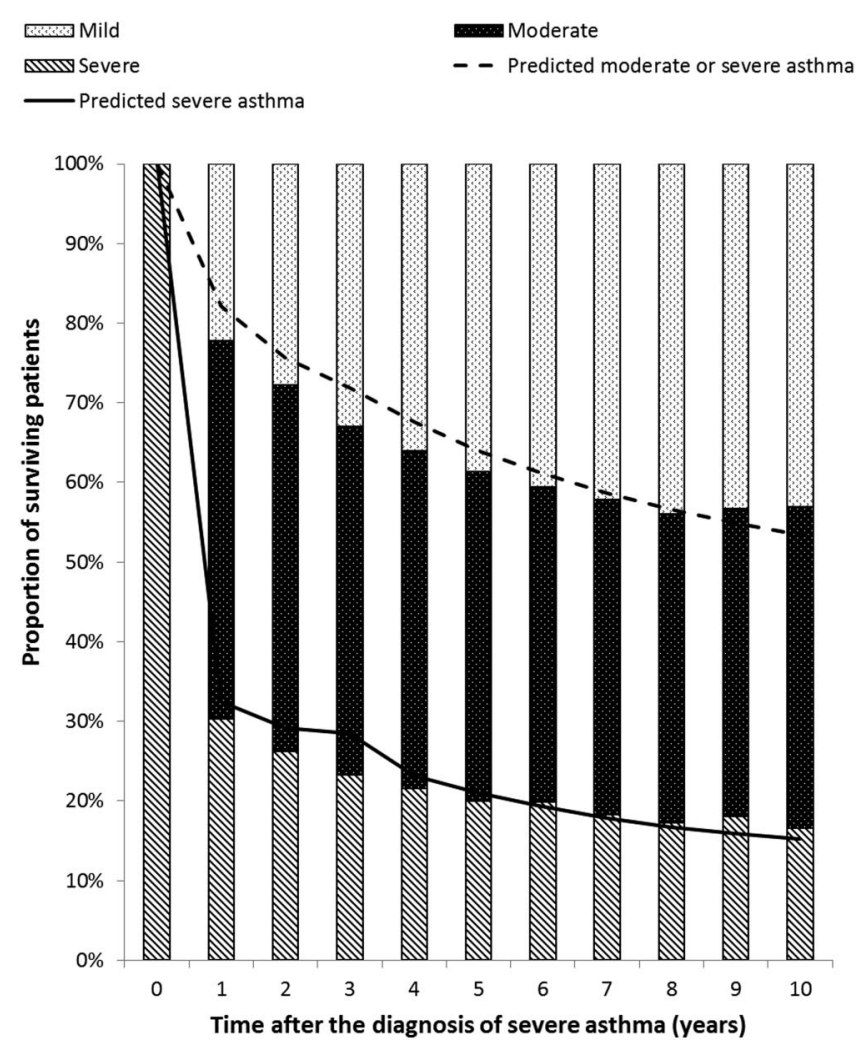

Figure 1 Proportions of surviving patients with mild, moderate and severe asthma over time. Bars indicate observed frequencies in the study cohort; lines indicate predicted population-level distributions from the regression model. 
those who met a validated definition of severe asthma in any year after at least 2 years of being classified as having non-severe or no asthma (see next section, 'Classification of asthma severity'). All included patients were followed until death, last date of registration with the programme or the end of available data (31 December 2012), whichever came first. The index year was defined as the first calendar year in which the patient was identified as having severe asthma. The unit of observation was patient-year. For each patient-year of data we assessed the level of severity and whether the patient died during that year. To reliably evaluate severity, we removed patient-years in which the patient was registered with the provincial health insurance programme for fewer than 300 days, except for the year in which they died.

\section{Classification of asthma severity}

We used a validated algorithm to classify each patient-year into three severity states (mild, moderate or severe) based on a combination of three variables: the intensity of controller therapy, the use of rescue medications and markers of moderate-severe exacerbations (ie, a filled prescription for oral corticosteroids, an emergency room visit and/or hospital admission for asthma). ${ }^{11}$ This algorithm was developed using Canadian databases and validated against the Canadian Asthma Consensus Guidelines. ${ }^{10}$ A resource use pattern consistent with severe asthma corresponded to the use of high doses of inhaled corticosteroids (ICS) in beclomethasone-chlorofluorocarbon equivalent (average daily dose of at least $1000 \mu \mathrm{g} /$ day) or reliever medications (more than 10 doses of short-acting $\beta 2$ agonists (SABAs) per week) while still experiencing moderate to severe exacerbations. A pattern consistent with mild asthma corresponded to low ICS doses $(0-250 \mu \mathrm{g} /$ day if receiving additional controller therapy, otherwise $0-500 \mu \mathrm{g} / \mathrm{day})$, without the occurrence of moderate to severe exacerbation or SABA use for more than three doses per week. A patient-year that did not meet the criteria for either severe or mild asthma was classified as moderate asthma. Additionally, a fourth state representing death was assigned to all patient-years in which death occurred.

\section{Assessment of risk factors}

We considered age, sex, socioeconomic status (SES), comorbidity and proportion of days covered (PDC) by controller medications as risk factors that could potentially affect the course of severe asthma. All these variables were ascertained in the index year. SES was categorised into three levels (low, middle, high) based on the median neighbourhood household income quintile, with low SES defined as being in the lowest two quintiles and high SES as the highest two quintiles. This variable is frequently used to study the effects of SES on health and healthcare expenditures and correlates well with individual-level SES. ${ }^{20}$ Comorbidity was quantified using a modified Charlson comorbidity index (CCI-excluding asthma from the score). ${ }^{21}$ Based on commonly used cut-off points in previous studies, ${ }^{22}$ comorbidity burden was classified into four ordinal levels: level 1 , CCI score $=0$; level 2 , score $=1$; level 3 , score $=2$; level 4 , score $\geq 3$, with higher levels corresponding to greater comorbidity. PDC was calculated as the total number of days with possession of any of the following commonly prescribed controller medications in the index year: systemic corticosteroids, ICS, ICS/long-acting $\beta 2$ agonists or leukotriene receptor antagonists (see online supplementary appendix 1). PDC was classified into three levels: level $1, \mathrm{PDC}<50 \%$; level 2 , PDC $\geq 50-80 \%$; level $3, \mathrm{PDC} \geq 80 \%$. $^{23}$

\section{Statistical analysis}

All statistical analyses were performed using SAS (V.9.3, SAS Institute Inc., Carey, North Carolina, USA). Online supplementary appendix 2 provides detailed description of the regression and computation methods.

We used a Markov model to examine the long-term trajectory of severity following incident severe asthma and the influences of baseline risk factors. A similar approach has been

Table 2 Adjusted OR of transition to different severity states

\begin{tabular}{|c|c|c|c|c|c|c|}
\hline \multirow{3}{*}{$\begin{array}{l}\text { Risk factors in the } \\
\text { index year }\end{array}$} & \multicolumn{6}{|c|}{ Severity state in any future year } \\
\hline & \multicolumn{2}{|c|}{$\begin{array}{l}\text { Transition to moderate/severe/death } \\
\text { vs mild }\end{array}$} & \multicolumn{2}{|c|}{$\begin{array}{l}\text { Transition to severe/death } \\
\text { vs mild/moderate }\end{array}$} & \multicolumn{2}{|l|}{$\begin{array}{l}\text { Transition to death } \\
\text { vs all other levels }\end{array}$} \\
\hline & OR $(95 \% \mathrm{Cl})$ & $\overline{p \text { Value }}$ & OR $(95 \% \mathrm{Cl})$ & $\overline{p \text { Value }}$ & OR $(95 \% \mathrm{Cl})$ & $\bar{p}$ Value \\
\hline Age & 1.01 (1.01 to 1.01$)$ & $<0.0001$ & 1.01 (1.01 to 1.01$)$ & $<0.0001$ & 1.05 (1.04 to 1.06$)$ & $<0.0001$ \\
\hline \multicolumn{7}{|l|}{ Sex } \\
\hline Female & Reference & & & & & \\
\hline Male & 1.12 (1.07 to 1.16$)$ & $<0.0001$ & 1.01 (0.96 to 1.05$)$ & 0.812 & 1.73 (1.41 to 2.12$)$ & $<0.0001$ \\
\hline \multicolumn{7}{|l|}{ SES } \\
\hline Low & Reference & & & & & \\
\hline Middle & $0.98(0.93$ to 1.04$)$ & 0.508 & $0.94(0.89$ to 1.00$)$ & 0.059 & $0.59(0.44$ to 0.79$)$ & 0.000 \\
\hline High & 0.98 (0.94 to 1.03$)$ & 0.445 & 0.88 (0.84 to 0.93$)$ & $<0.0001$ & $0.56(0.44$ to 0.72$)$ & $<0.0001$ \\
\hline \multicolumn{7}{|l|}{ Comorbidity } \\
\hline $\mathrm{CCl}$ score $=0$ & Reference & & & & & \\
\hline $\mathrm{CCl}$ score $=1$ & 1.05 (0.99 to 1.12$)$ & 0.101 & 1.08 (1.01 to 1.16$)$ & 0.020 & $0.72(0.54$ to 0.96$)$ & 0.025 \\
\hline $\mathrm{CCl}$ score $=2$ & $1.11(0.99$ to 1.24$)$ & 0.080 & $1.32(1.17$ to 1.49$)$ & $<0.0001$ & 1.94 (1.32 to 2.87$)$ & 0.001 \\
\hline $\mathrm{CCl}$ score $\geq 3$ & 1.05 (0.92 to 1.19$)$ & 0.466 & $1.48(1.29$ to 1.7$)$ & $<0.0001$ & 4.24 (2.96 to 6.09$)$ & $<0.0001$ \\
\hline \multicolumn{7}{|c|}{ PDC of asthma controller medications } \\
\hline PDC $<50 \%$ & Reference & & & & & \\
\hline $50 \% \leq \mathrm{PDC}<80 \%$ & 1.07 (1.02 to 1.12$)$ & 0.008 & 1.22 (1.16 to 1.29$)$ & $<0.0001$ & 1.10 (0.88 to 1.39$)$ & 0.400 \\
\hline $\mathrm{PDC} \geq 80 \%$ & $1.20(1.11$ to 1.3$)$ & $<0.0001$ & $1.26(1.17$ to 1.35$)$ & $<0.0001$ & $0.87(0.62$ to 1.22$)$ & 0.414 \\
\hline
\end{tabular}


used to model the trajectory of asthma control. ${ }^{24} 25$ This approach models longitudinal severity patterns as a sequence of transitions between severity states over time. We modelled severity in the next year based on severity history in the current and past 2 years. The use of 3-year history was required to satisfy the Markovian property, that is, the likelihood of transition to a future disease state depended only on the disease states in the current and past 2 years, not on any history before the past 2 years. ${ }^{26}$ This property makes Markov models a powerful tool in studying disease trajectories as it enables long-term projections of disease states under the influences of risk factors.

We estimated the transition matrix of this Markov process using an ordinal logistic regression with severity state $(0=$ mild, $1=$ moderate, 2 =severe, $3=$ death) in the next year as the dependent variable, and severity history in the current and the past 2 years as independent variables (see online supplementary appendix 3 for a sample transition matrix). The aforementioned baseline risk factors were included as covariates of interests to assess their independent adjusted effects on the probability of transition between severity states. We further adjusted for calendar effect by including the calendar year of the index year. This model was fitted using a generalized linear model with generalized estimating equations to account for the longitudinal observations of patient-years within individuals. ${ }^{27}$ The effect of each independent variable was estimated in terms of three ORs of transition from a past severity state to a given severity state in the next year: transition to moderate/severe/death versus transition to mild, transition to severe/death versus transition to mild/ moderate, and transition to death versus transition to mild/moderate/severe.

To assess model fit, we compared the observed versus predicted population-averaged trajectories of severity after incident severe asthma over the entire study period. The disease trajectory was defined as the probability of being in a particular severity state at a given follow-up year over the next 10 years. Once
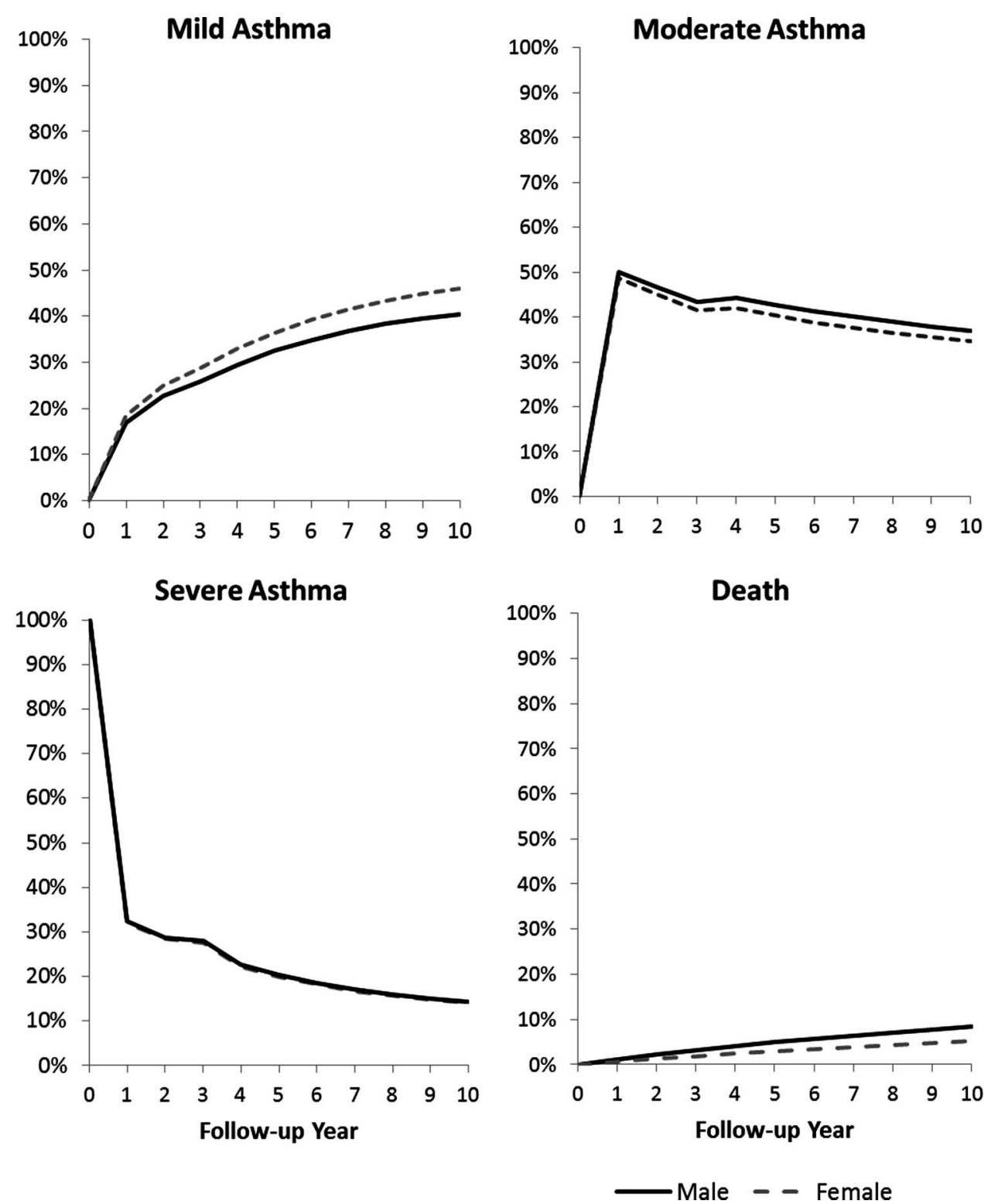

Figure 2 The influence of sex on long-term trajectory of severe asthma. Plotted curves show adjusted probability of being in different severity states in the following 10 years since the index year, stratified by sex. Each graph corresponds to a different severity state: (A) mild asthma, (B) moderate asthma, (C) severe asthma, (D) death. 
the model fit was assured, we estimated the trajectories of severe asthma as a function of a given baseline risk factor, while adjusting for all other risk factors.

\section{RESULTS}

Of 285287 patients $14-55$ years of age who met the initial case definition of asthma, we identified 13467 (5\%) with a new onset of severe asthma. Table 1 presents the characteristics of this sample in the index year. The mean age was 36.8 years and $55 \%$ were female. The average follow-up was 5.5 years (see online supplementary appendix 4 for the proportions of patients remaining in the cohort during the follow-up period). Approximately $47 \%$ of the sample was classified as having low SES and $85 \%$ had at least one comorbid condition. The majority $(76 \%)$ experienced at least one moderate to severe exacerbation in their index year; only $10 \%$ had PDC of $80 \%$ or more (table 1).
While all patients were classified as having severe asthma in the index year, the proportion of patients remaining in the severe state decreased consistently in the first 4 years and nearly plateaued in the next 6 years (figure 1). Ten years after the onset of severe asthma, $394(3 \%)$ patients had died; of the patients still on study, $43 \%, 40 \%$ and $17 \%$ were classified as having mild, moderate and severe asthma, respectively (figure 1). The predicted trajectory of severity from the regression model aligned well with the observed clinical course of severe asthma (figure 1).

Table 2 presents the association between risk factors in the index year on transition between severity states. Older age was associated with higher likelihood of being in more severe states in the future. Compared with women, men were less likely to transition to mild asthma, but were more likely to die; their chances of remaining in severe asthma were the same. Greater comorbidity in the index year was associated with higher
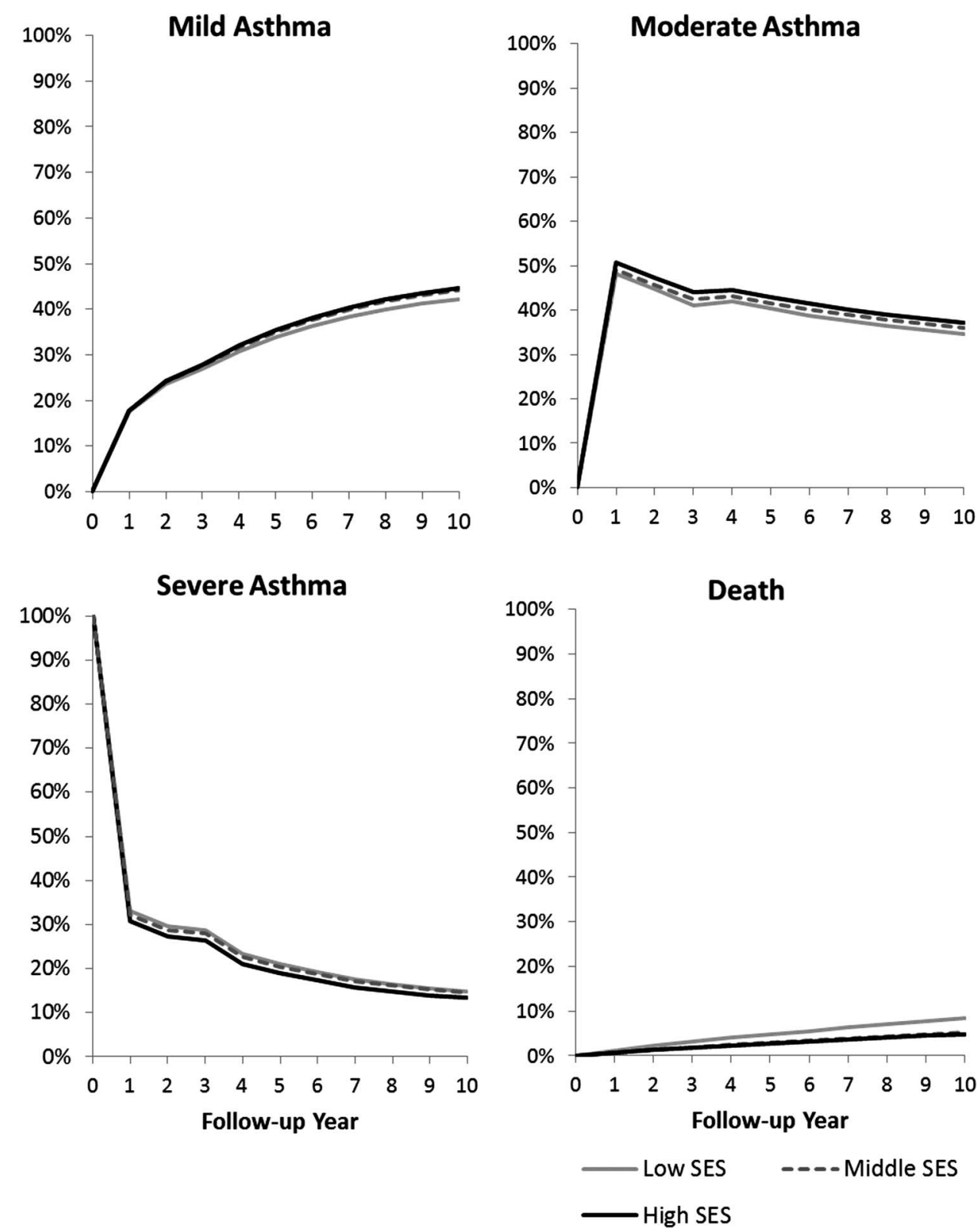

Figure 3 The influence of baseline socioeconomic status (SES) on long-term trajectory of severe asthma. Plotted curves show adjusted probability of being in different severity states in the following 10 years since the index year, stratified by baseline SES. Each graph corresponds to a different severity state: (A) mild asthma, (B) moderate asthma, (C) severe asthma, (D) death. 
likelihood of death or otherwise remaining in severe asthma, whereas high SES was associated with lower likelihood of such transitions. Having greater PDC was positively associated with the likelihood of moderate and severe asthma in the future. Online supplementary appendix 5 shows the full regression analysis results.

Figures 2-5 show how risk factors in the index year affected the 10 -year trajectory of severe asthma. There were few sexrelated differences in the long-term probability of leaving the severe state (figure 2). Compared with high SES, low SES was associated with a $10 \%$ increase in patient time with severe asthma over the next 10 years (figure 3 ). Higher comorbidity burden was associated with more patient time in the severe state (CCI score=1 vs 0: $13 \%$, CCI score $\geq 2$ vs 0: $24 \%$ increase in person-time in severe asthma within 10 years) (figure 4). Compared with PDC of less than 50\%, PDC between 50\% and $80 \%$ and PDC of $80 \%$ or more were associated with $25 \%$ and
$35 \%$ increased patient time in severe asthma in the next 10 years, respectively (figure 5). Male sex, low SES and high comorbidity were associated with a higher 10-year risk of death, with comorbidity having the most prominent impact (figures 2-4).

\section{DISCUSSION}

We developed a multi-state Markov model to quantify the longterm trajectory of asthma severity and the influence of risk factors that were known early in the clinical course. The validity of this model was assessed by comparing the predicted and observed trajectories which showed that it fitted the data well. We found that the majority of patients who were classified as severe in the first year according to their asthma-related resource use patterns transitioned to less severe states in the subsequent years. High SES, lower comorbidity burden and PDC of less than $50 \%$ in the first year were independently associated with a better prognosis, while sex was not.
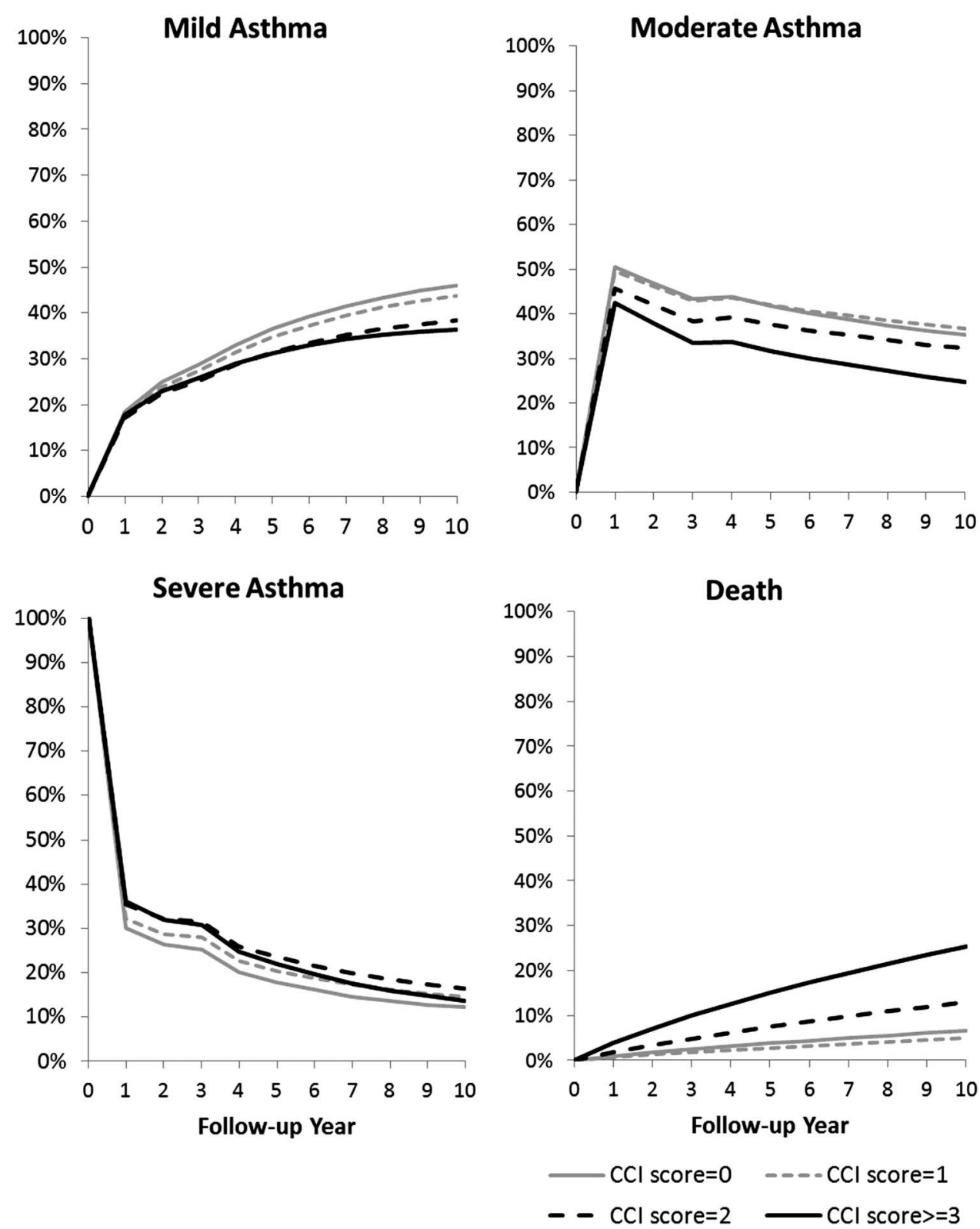

Figure 4 The influence of baseline comorbidity burden on long-term trajectory of severe asthma. Plotted curves show adjusted probability of being in different severity states in the following 10 years since the index year, stratified by baseline Charlson Comorbidity Index (CCI) classification. Each graph corresponds to a different severity state: (A) mild asthma, (B) moderate asthma, (C) severe asthma, (D) death. 

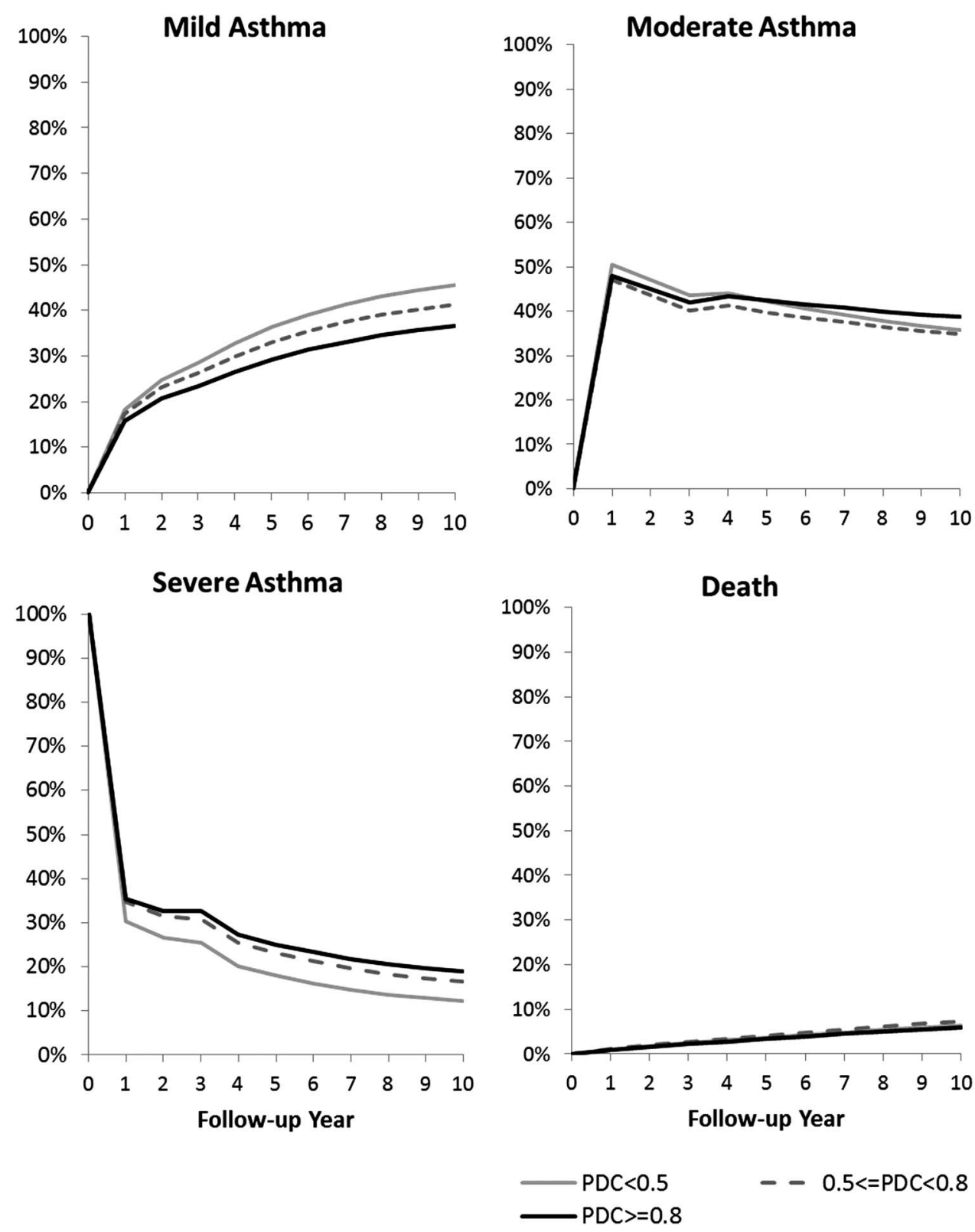

Figure 5 The influence of proportion of days covered (PDC) by asthma controller therapy in the index year on long-term trajectory of severe asthma. Plotted curves show adjusted probability of being in different severity states in the following 10 years since the index year, stratified by baseline PDC levels. Each graph corresponds to a different severity state: (A) mild asthma, (B) moderate asthma, (C) severe asthma, (D) death.

There are likely many influences that can cause an inception cohort of severe asthma to transition to non-severe asthma over time. For instance, the phenomenon referred to as 'regression to the mean' indicates that incident new onset of severe asthma may be a temporary state. ${ }^{28}$ The extent of this phenomenon is affected by the intrinsic variability of disease activity. In this cohort, the probability of staying classified as severe considerably decreased in the first 4 years and then remained relatively stable. Our findings are consistent with the study by Ernst et $a l^{12}$ which showed a similar course for severe asthma, with $61 \%$ of the decrease in treatment intensity (interpreted as an indicator of non-severe asthma) occurring within the first 5 years.

In terms of risk factors, our study confirmed previous findings that there was no sex-related difference in 'outgrowing' severe asthma. $^{12} 28$ Additionally, to the best of our knowledge, this is the first time that independent adverse impacts of low SES and high comorbidity on the long-term course of severe asthma has been demonstrated. There are multiple paths by which SES could affect the prognosis of severe asthma. Low SES is associated with a combination of environmental and individual risk factors of asthma symptoms and exacerbations, including residential and occupational exposure to asthma triggers, tobacco smoke and knowledge about asthma management. ${ }^{29-32}$ Canada has a universal health care system. Although there are few SES-related barriers to access to and continuity of care, ${ }^{33} 34$ low SES is still associated with inappropriate medication management, such as overuse of reliever medications and underuse of controller medications. ${ }^{35}$ In addition, patients with low SES and high comorbidity have poorer overall health, which might impede the remission of severe asthma. ${ }^{29} 36$ Likewise, many chronic conditions and their treatments affect the response to asthma treatments or increase difficulty in achieving asthma control. $^{37} 38$ 
Although patients with higher PDC of controller therapy in the first year were found to have a longer period of severe asthma, this may be due to residual confounding by disease severity. This is because PDC is both a measure of adherence and an indicator of treatment intensity, and treatment intensity plays a central role in the classification of asthma severity. ${ }^{11}$ The residual variation in disease severity among individuals that are classified as having severe asthma might result in spurious correlation between treatment intensity and adverse future outcomes.

This study is the first to examine the long-term natural history of severe asthma and influences of multiple risk factors at diagnosis on the subsequent course of the disease. Earlier natural history studies predominantly focused on decline in lung function. ${ }^{5-8}$ While these studies improved the understanding of the underlying phenotypes, their findings cannot directly translate to clinical and policy aspects such as the intensity of resource use. Here we classified levels of severity based on the intensity of treatment (current impairment) and the presence of exacerbation and death (future risk), ${ }^{11}$ two fundamental and clinically relevant dimensions of severity advocated by clinical guidelines. $^{8}$

A major strength of this study is that it is based on a large population sample of patients in a well defined geographic region, and thus, has a low risk of selection bias. The real-world population-based nature of this study adds to the external validity and generalisability of our results. In addition, our robust and powerful statistical approach, based on principles previously developed and validated in asthma, ${ }^{39}$ enabled us to convert measures of relative effect such as OR to more straightforward metrics representing the impact of early risk factors on disease trajectories. However, the limitations of this study should be considered. First, without access to objective measures such as lung function and patients' symptoms, the definition of severity from administrative data can only approximate the clinical definition. Thus, the findings of this study should be considered together with clinical judgement at the time of individual patient care. Second, as the algorithm we used to classify levels of severity was partly based on the intensity of prescription drugs, ${ }^{11}$ it is possible to misclassify patient-years into a milder state when patients had severe disease but less inclined to use asthma medications (or used alternative and complementary medicines), for example, during pregnancy. It is also possible to misclassify milder patient-years into more severe states because information regarding asthma medications in the administrative data represents the filled prescriptions, but not necessarily the actual consumption of medication. However, since the measurement of severity was based on medication use and on markers of exacerbations, ${ }^{11}$ the chance of a severe patient-year being misclassified as mild was low; rather, there was a greater likelihood that a patient-year with mild asthma was misclassified as severe. Thus, any misclassification bias in our study would likely lead to an underestimation of the proportion of patients who eventually transitioned to non-severe asthma. Finally, due to lack of access to external data we could not externally validate the Markov transition cohort. Such external validation could add to the utility of the presented framework and enable prediction of future disease burden in other patient populations based on their risk factor profile.

In conclusion, our study found that incident severe asthma was generally not progressive because most patients transitioned to milder states. Predictors of poor long-term prognosis included low SES and high comorbidity, whereas there were few sex-related differences in the disease course. These results indicate the potentially modifiable course of severe asthma. Current asthma management guidelines emphasise achieving and maintaining asthma control, ${ }^{40}$ and the effectiveness of such guidelines is mainly established by the concurrent relationship between asthma control and lower burden of asthma. ${ }^{41}$ However, programmes and interventions with the ability to modify the long-term trajectory of asthma will confer farreaching benefits and affect the benefit-risk profile of asthma management guidelines. Future studies can build on this methodology to expand our understanding of the effects of modifiable risk factors and interventions on the long-term prognosis of asthma and its future burden.

Contributors WC conceptualised and designed the study, developed the analytical framework, cleaned and prepared the data, performed statistical analysis and wrote the first draft of the manuscript. MS conceptualised the research idea and assisted in the study design. MS and ZZ contributed to the statistical analysis. CAM, LDL and JMF were involved in the acquisition of the data. CAM, LDL and JMF contributed to the interpretation of findings, provided interim feedback during data analysis and contributed to the study design. JMF provided clinical judgement that affected study design and interpretation of findings. CAM, LDL, JMF, MS and ZZ critically revised the manuscript. All authors approved the final version of the manuscript.

Funding This work was supported by AllerGen National Centre of Excellence as part of the project 'Platform for Outcomes Research and Translation in Asthma and Allergy (PORTAL)', and the health economics platform of the Canadian Respiratory Research Network (CRRN); the National Sanatorium Association (to MS); and a tier 2 Canada Research Chair in Pharmaceutical Outcomes (to CAM).

Competing interests None declared.

Ethics approval University of British Columbia Human Ethics Certificate H08-01287.

Provenance and peer review Not commissioned; externally peer reviewed.

\section{REFERENCES}

1 Braman SS. The global burden of asthma. Chest 2006;130(Suppl 1):4S-12S.

2 Bedouch P, Marra CA, FitzGerald JM, et al. Trends in asthma-related direct medical costs from 2002 to 2007 in British Columbia, Canada: a population based-cohort study. PLOS ONE 2012;7:e50949.

3 Bahadori K, Doyle-Waters MM, Marra C, et al. Economic burden of asthma: a systematic review. BMC Pulm Med 2009;9:24

4 Lazarus SC, Boushey HA, Fahy JV, et al. Long-acting beta2-agonist monotherapy vs continued therapy with inhaled corticosteroids in patients with persistent asthma: a randomized controlled trial. JAMA 2001;285:2583-93.

5 Moore WC, Bleecker ER, Curran-Everett D, et al. Characterization of the severe asthma phenotype by the National Heart, Lung, and Blood Institute's Severe Asthma Research Program. J Allergy Clin Immunol 2007;119:405-13.

6 Sorkness RL, Bleecker ER, Busse WW, et al. Lung function in adults with stable but severe asthma: air trapping and incomplete reversal of obstruction with bronchodilation. J Appl Physiol (1985) 2008;104:394-403.

7 Matsunaga K, Akamatsu K, Miyatake A, et al. Natural history and risk factors of obstructive changes over a 10-year period in severe asthma. Respir Med 2013:107:355-60.

8 National Asthma Education and Prevention Program, Third Expert Panel on the Diagnosis and Management of Asthma. Expert Panel Report 3: Guidelines for the Diagnosis and Management of Asthma. Bethesda, MD: National Heart, Lung, and Blood Institute (US) (Internet). 2007. http://www.ncbi.nlm.nih.gov/books/ NBK7232/

9 Cockcroft DW, Swystun VA. Asthma control versus asthma severity. J Allergy Clin Immunol 1996;98:1016-8.

10 Boulet L-P, Becker A, Bérubé D, et al. Canadian asthma consensus report, 1999. Can Med Assoc J 1999;161(11 Suppl 1):S1-5.

11 Firoozi $\mathrm{F}$, Lemière $\mathrm{C}$, Beauchesne $\mathrm{M}-\mathrm{F}$, et al. Development and validation of database indexes of asthma severity and control. Thorax 2007;62:581-7.

12 Ernst $\mathrm{P}, \mathrm{Cai} B$, Blais $\mathrm{L}$, et al. The early course of newly diagnosed asthma. Am J Med 2002;112:44-8.

13 Government of Canada Statistics Canada. Population by sex and age group, by province and territory (number, both sexes). 2014 (cited 6 Feb 2015). http://www. statcan.gc.ca/tables-tableaux/sum-som/101/cst01/demo31a-eng.htm

14 British Columbia Ministry of Health (2013). Consolidation File (MSP Registration \& Premium Billing). Population Data BC. Data BC. Data Extract. MOH, 2014. http:// www.popdata.bc.ca/data

15 BC Vital Statistics Agency (2013). Vital Statistics Deaths. Population Data BC. Data Extract. BC Vital Statistics Agency, 2014. http://www.popdata.bc.ca/data 
16 British Columbia Ministry of Health (2014). Discharge Abstract Database (Hospital Separations). Population Data BC. Data Extract. MOH, 2014. http://www.popdata. bc.ca/data

17 British Columbia Ministry of Health. Medical Services Plan (MSP) payment information file. Population Data BC. Data Extract. MOH, 2014. http://www. popdata.bc.ca/data

18 British Columbia Ministry of Health (2013). PharmaNet. BC Ministry of Health. Data Extract. Data Stewardship Committee, 2013. http://www.popdata.bc.ca/data

19 Canadian Institute for Health Information. CIHI Data Quality Study of the DAD 2009-2010 Discharge Abstract Database. 2012 (cited 1 Apr 2015). https://secure. cihi.ca/estore/productFamily.htm?pf=PFC1762\&lang=en\&media $=0$

20 Gan WQ, FitzGerald JM, Carlsten C, et al. Associations of ambient air pollution with chronic obstructive pulmonary disease hospitalization and mortality. Am J Respir Crit Care Med 2013;187:721-7.

21 Charlson ME, Pompei P, Ales KL, et al. A new method of classifying prognostic comorbidity in longitudinal studies: development and validation. J Chronic Dis 1987:40:373-83.

22 Núñez JE, Núñez E, Fácila L, et al. [Prognostic value of Charlson comorbidity index at 30 days and 1 year after acute myocardial infarction]. Rev Esp Cardiol 2004;57:842-9.

23 Karve S, Cleves MA, Helm M, et al. Good and poor adherence: optimal cut-point for adherence measures using administrative claims data. Curr Med Res Opin 2009;25:2303-10.

24 Combescure $C$, Chanez $P$, Saint-Pierre $P$, et al. Assessment of variations in control of asthma over time. Eur Respir J 2003;22:298-304.

25 Bateman ED, Reddel HK, Eriksson G, et al. Overall asthma control: the relationship between current control and future risk. J Allergy Clin Immunol 2010;125:600-8, 608.e1-e6.

26 Feller W. An introduction to probability theory and its applications. Vol II. New York: John Wiley \& Sons, 1971.

27 Liang K-Y, Zeger SL. Longitudinal data analysis using generalized linear models. Biometrika 1986;73:13-22.
28 Panhuysen $\mathrm{Cl}$, Vonk JM, Koëter $\mathrm{GH}$, et al. Adult patients may outgrow their asthma: a 25-year follow-up study. Am J Respir Crit Care Med 1997;155:1267-72.

29 Adler NE, Ostrove JM. Socioeconomic status and health: what we know and what we don't. Ann N Y Acad Sci 1999;896:3-15.

30 Novotny TE, Warner KE, Kendrick JS, et al. Smoking by blacks and whites: socioeconomic and demographic differences. Am J Public Health 1988;78:1187-9.

31 Evans GW, Kantrowitz E. Socioeconomic status and health: the potential role of environmental risk exposure. Annu Rev Public Health 2002;23:303-31.

32 Poureslami IM, Rootman I, Balka E, et al. A systematic review of asthma and health literacy: a cultural-ethnic perspective in Canada. Medscape Gen Med 2007;9:40.

33 Erzen D, Carriere KC, Dik N, et al. Income level and asthma prevalence and care patterns. Am J Respir Crit Care Med 1997;155:1060-5.

34 Roos LL, Walld R, Uhanova J, et al. Physician visits, hospitalizations, and socioeconomic status: ambulatory care sensitive conditions in a Canadian setting. Health Serv Res 2005;40:1167-85.

35 Anis $A H$, Lynd LD, Wang $X$, et al. Double trouble: impact of inappropriate use of asthma medication on the use of health care resources. CMAJ Can Med Assoc J 2001;164:625-31.

36 Merikangas KR, Ames M, Cui L, et al. The impact of comorbidity of mental and physical conditions on role disability in the US adult household population. Arch Gen Psychiatry 2007;64:1180-8.

37 Boulet L-P. Influence of comorbid conditions on asthma. Eur Respir J 2009;33:897-906.

38 Xuan J, Kirchdoerfer LJ, Boyer JG, et al. Effects of comorbidity on health-related quality-of-life scores: an analysis of clinical trial data. Clin Ther 1999;21:383-403.

39 Austin PC, Urbach DR. Using G-computation to estimate the effect of regionalization of surgical services on the absolute reduction in the occurrence of adverse patient outcomes. Med Care 2013;51:797-805.

40 Global Initiative for Asthma. GINA Report, Global Strategy for Asthma Management and Prevention (cited 4 Jul 2014). http://www.ginasthma.org/documents/4

41 Centers for Disease Control and Prevention. Asthma-CDC's National Asthma Control Program (cited 1 Oct 2015). http://www.cdc.gov/asthma/nacp.htm 